Paroxysmal nonepileptic events in children and adolescents vary with age (Kotagal $\mathrm{P}$ et al. Pediatrics 2002;110(4):e46). 1) Infant, Toddler, and Preschool Group (2 mos - 5 years; 26 patients), the most common diagnoses were stereotyped movements, hypnic jerks, parasomnias, and Sandifer syndrome (spasmodic torticollis, hiatus hernia, and gastroesophageal reflux). Concomitant epilepsy was present in 12 patients (46\%); 2) The School-Age Group (5 - 12 years; 61 patients), Most common diagnoses were conversion disorder (psychogenic seizures), inattention or daydreaming, stereotyped movements, hypnic jerks, and paroxysmal movement disorders. Concomitant epilepsy in 15 patients (25\%); 3) The Adolescent Group (12 - 18 years) 48 patients of whom $40(83 \%)$ were diagnosed with conversion disorder. Nine $(19 \%)$ had concomitant epilepsy. Conversion disorder was most common in the adolescent group and in females, whereas males predominated in the school-age group. Concomitant epilepsy with nonepileptic events occurred in all age groups.

\title{
BENIGN INFANTILE SEIZURES (FUKUYAMA SYNDROME)
}

Benign infantile seizures (BIS), first described by Fukuyama (1963) as generalized convulsions were later reported as focal or partial seizures, mainly nonfamilial, and more recently as familial with autosomal dominance. The two types, familial and non-familial, generalized or focal, are now presented as one syndrome of Fukuyama, Watanabe, and Vigevano. The occurrence of the syndrome in Saudi Arabia is reported by researchers at King Fahad Military Hospital, and King Saud University. Inclusion criteria for BIS were as follows: 1) age of onset 2-24 months; 2) normal development; 3) normal interictal EEG; 4) normal brain imaging; and 5) good response to treatment. Of 116 infants, between 2 and 24 months, with epilepsy, 14 (12\%) showed electroclinical features consistent with BIS; 11 patients fulfilled criteria for benign nonfamilial infantile seizures (BNFIS), and 3 had pedigrees consistent with benign familial infantile seizures (BFIS). All responded to anticonvulsants, and 50\% responded within 3 months. (Saardeldin IY, Housawi Y, Al Nemri A, Al Hifzi I. Benign familial and nonfamilial infantile seizures (Fukuyama-Watanabe-Vigevano syndrome): A study of 14 cases from Saudi Arabia. Brain Dev May 2010;32:378-384). (Respond: Dr IY Saardeldin.E-mail: eysaad@yahoo.co.uk).

COMMENT. First described in Japan, the syndrome of BIS is now reported in other countries, including Saudi Arabia. In the new classification described below, BIS or benign infantile epilepsy is included under the electroclinical syndromes defined by age, but grouped with West syndrome and Dravet syndrome, both having a poor prognosis.

\section{ILAE REVISED CLASSIFICATION AND TERMINOLOGY OF SEIZURES AND EPILEPSIES, 2005-2009}

The International League Against Epilepsy (ILAE) Commission on Classification and Terminology has revised concepts, terminology, and approaches for classifying seizures and forms of epilepsy, last updated in 1981 for seizures and in 1989 for epilepsies. Changes in the 1981 classification for seizures include the following: 
1) Neonatal seizures are not a separate entity and are included under "electroclinical syndromes arranged by age at onset"; 2) Spasms are now recognized as "epileptic spasms," which includes infantile spasms; 3) Focal simple partial and complex partial categories of focal seizures are eliminated and replaced by focal "without impairment of consciousness or awareness" and "with impairment of consciousness or awareness;" 4) Focal seizure "evolving to a bilateral convulsive seizure" replaces the term "secondarily generalized seizure;" and 5) "Myoclonic atonic" replaces "myoclonic astatic" seizures. The new seizure classification has 3 main categories: generalized (tonic-clonic, absence, myoclonic, clonic, tonic, atonic), focal, and unknown. The new classification of electroclinical syndromes and other epilepsies is grouped according to 1) age at onset (neonatal period, infancy, childhood, and adolescent-adult), 2) as distinctive "constellations," (mesial temporal lobe epilepsy, Rasmussen syndrome, gelastic seizures, hemiconvulsion-hemiplegia-epilepsy); epilepsies attributed to "structural-metabolic" causes, to angioma, of unknown cause, and seizures not recognized as epilepsies (benign neonatal seizures and febrile seizures). The concepts of generalized and focal do not apply to electroclinical syndromes. Genetic, structural-metabolic, and unknown represent modified concepts to replace idiopathic, symptomatic, and cryptogenic. (Berg AT, Berkovic SF, Brodie MJ, et al. Revised terminology and concepts for organization of seizures and epilepsies: Report of the ILAE Commission on Classification and Terminology, 2005-2009. Epilepsia April 2010;51(4):676-685). (Respond: Dr Anne T Berg, Department of Biology, Northern Illinois University, DeKalb, IL 60115. E-mail: atberg@niu.edu).

COMMENT. As one of the last Fellows privileged to have known and worked under Doctor William Gordon Lennox at his Seizure Unit, Children's Medical Center, Boston, in 1953-1954, I am sure he would have applauded the attempts to reclassify seizures and advance our understanding of the epilepsies. In keeping with some of the editorial comments regarding the new classification (Epilepsia 2010;51(4):713-723), Dr Lennox might have questioned the need to replace the terms idiopathic, symptomatic, and cryptogenic. As intended, the ILAE Commission has provided a basis and guide for further epidemiological studies. In the last half century, I have witnessed many changes in the classification of seizures since the simple triad of grand mal, petit mal, and psychomotor, genetic or acquired, metabolic or organic (structural). In the use of the term structural-metabolic in the new classification we have partially reverted to the Lennox terminology. (Epilepsy and Related Disorders. Vol 1. Boston; Little, Brown and Company, 1960).

\section{HEADACHE DISORDERS}

\section{ACUTE CONFUSIONAL MIGRAINE RESPONSE TO IV VALPROATE}

The case of a 12-year-old girl with an attack of acute confusional migraine (ACM) that responded rapidly to intravenous valproic acid is reported from Meir Medical Center, Tel Aviv University, Israel. One hour before arriving at the ED, she complained of bilateral blurriness of her lower visual fields, frontal headache, and paresthesiae of left arm and 